\title{
A Multi-AUV Missions Simulation Framework for the USARSim Robotics Simulator
}

\author{
Anuj Sehgal, Daniel Cernea \\ Indian Underwater Robotics Society \\ E-118 Nar Vihar Part-I, Sector 34 \\ Noida 201301, India \\ \{anuj, daniel\}@iurs.org
}

\begin{abstract}
The maximum potential for underwater exploration rests within the use of multiple Autonomous Underwater Vehicles (AUVs) and tasks involving human diver-AUV coordination. Such tasks require dependable underwater wireless communications, which normally utilize the rapidly varying acoustic channel. Rigorous testing of underwater acoustic communication systems is necessary to develop a dependable network, however, the high offshore testing costs make this difficult. Though simulators could aid the development of such AUV communication systems, the few existing simulators focus upon simulating a single vehicle and, as such, do not provide tools for simulating underwater communication systems. In this paper we present an underwater communications simulation framework designed for the Unified System for Automation and Robotics Simulator (USARSim). This simulation tool is capable of modeling networked communications between, or with, AUVs by accurately characterizing the underwater acoustic channel. Details on this simulation framework are provided along with some results obtained during development of this tool.
\end{abstract}

\section{INTRODUCTION}

Off-shore research and exploration has seen an increased use of Autonomous Underwater Vehicles (AUVs) in the last few years. Using multiple AUVs to perform coordinated tasks or deploy AUVs in diver coordinated missions holds the maximum potential for the growing use of AUVs. However, the high cost of conducting off-shore missions has led to limited testing time for design and development of the cooperation algorithms and systems, thereby reducing the full potential of such systems. From fabrication to deployment the costs associated with multiple AUVs deployed in a network can be quite high. Coordination and control of multiple AUVs requires dependable wireless communications between each vehicle. Since the radio channel does not function underwater and the optical channel has very limited transmission range [1], most wireless underwater communications between AUVs are implemented using the underwater acoustic channel. The costs of a dependable underwater acoustic modem too are in the order of several thousand dollars and off shore deployment and recovery of underwater vehicles from a small boat can be in thousands per day [2]. Such high costs associated with off-shore testing can be a bane to development in case revisions are necessary.

Using the acoustic channel access method can enable multiple wireless network applications in the aquatic environment, but it poses unique challenges to achieve near real-time communications as a result of the channel characteristics. Limited bandwidth-capacity, low battery power availability with none to little possibility of recharging and the high likelihood of network disruptions [3] are just some of the problems posed by the oceanic environment. Issues such as long and varying propagation delays, multi-path echoes and high and varying ambient noise make the acoustic communication design process highly error-prone, and as such, must also be taken into consideration during the design of any underwater acoustic communication system.

Channel complexity and the high costs associated with off-shore deployment and testing highlight the need for a simulator that can accurately model the effects of the underwater environment on AUVs and the effects of the volatile underwater acoustic communication channel used to implement the coordination and control data exchange.

Not much work has gone into simulating the underwater environment in the popular robotics simulators [4]. Furthermore, the effort in simulating network communication between AUVs has also been severely lacking [5], thereby forcing system designers to depend upon off-shore testing for dependable results. The Unified System for Automation and Robotics Simulator (USARSim) environment is quite popular with the rescue robotics community due to its strong physics engine and the ability to easily create models of new environments and robots. The extensibility of USARSim combined with its proven capabilities in simulating multi-robot cooperative tasks makes it an ideal simulation environment. As a result of its physics engine base, and model import capabilities, USARSim already has the ability to model the underwater environment and simple marine vehicles. However, the ocean being a highly complex medium for the propagation of sound, due to inhomogeneities and random fluctuations, including effects of the rough seas and ocean bottom variances, it is necessary to build extensions that would provide feedback on acoustic communications. The Wireless Simulation Server (WSS) underwater acoustic communications plugin for USARSim was designed for this purpose.

Following a discussion on the acoustic propagation models, the channel characterization approach adopted for developing the WSS plugin is discussed in this paper. An overview of the USARSim robotics simulator and the extensions we 
TABLE I

VALUES FOR REPRESENTING TYPES OF GEOMETRICAL SPREADING VIA THE GEOMETRICAL SPREADING COEFFICIENT $k$

\begin{tabular}{|c|c|c|c|}
\hline & Spherical & Cylindrical & Practical \\
\hline \hline$k$ & 2 & 1 & 1.5 \\
\hline
\end{tabular}

made to it in order to enable simulations of multiple AUV missions are also provided. Following a discussion on the acoustic propagation models, the channel characterization approach adopted for developing the WSS plugin is discussed in this paper. An overview of the USARSim robotics simulator and the extensions we made to it in order to enable simulations of multiple AUV missions are also provided. A discussion on the extensions to WSS for enabling underwater simulations and also some results based on our test cases is followed by conclusions.

\section{Acoustic Propagation Model}

The acoustic channel is considerably different from the commonly used radio channel [6]. As such, it is important to develop a complete channel model that can be used in simulation work. In this section we present the different aspects of the underwater acoustic channel as they were used in our simulation model.

\section{A. Propagation Delay}

The underwater channel is a complex environment which is effected by many varying factors, primarily temperature, salinity and depth. While taking the speed of sound in water to be $1500 \mathrm{~m} / \mathrm{s}$ is accurate within a certain range, a better approximation of this value is needed in order to model the effects of the aquatic environment on acoustic communications, since each of the aforementioned factors may also be interdependent or varying across the ocean.

The speed of sound in water has been a focus of analysis by many mathematical models. We chose to utilize the expression proposed by the authors of [7] since it calculates the speed of sound in water with an error in the speed estimate in the range of approximately $0.070 \mathrm{~m} / \mathrm{s}$ :

$$
\begin{aligned}
v= & 1448.96+4.591 C-5.304 \cdot 10^{-4} C^{3} \\
& +1.340(S-35)+1.630 \cdot 10^{-2} D+1.675 \cdot 10^{-7} D^{2} \\
& -1.025 \cdot 10^{-2} C(S-35)-7.139 \cdot 10^{-13} C D^{3}
\end{aligned}
$$

where $v$ is the sound velocity in $\mathrm{m} / \mathrm{s}, C$ is the temperature in degrees Celsius, $S$ is the salinity in parts per trillion (ppt) and $D$ is the depth in meters.

Since the speed of sound can vary greatly in regions of thermocline and halocline, and most AUV missions operate within these regions [8], determining speed of sound accurately is crucial.

\section{B. Transmission Loss}

The transmitted acoustic signal between AUVs reduces in overall signal strength over distance due to a host of factors governing sound propagation factors in the ocean. This decrease of acoustic intensity between the source and
TABLE II

FISHER \& SIMMONS' MODEL COEFFICIENTS

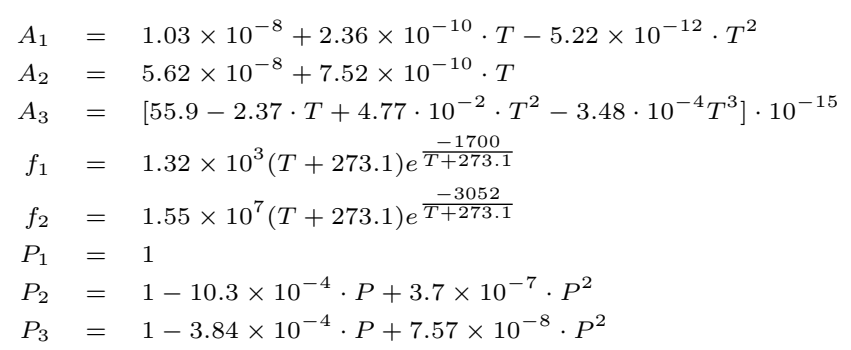

receiver, termed transmission loss, is composed majorly of three aspects, namely, geometrical spreading, attenuation and the anomaly of propagation. Geometrical spreading deals with the signal losses that occur due to focusing and defocusing effects caused by spreading of acoustic waves in the ocean water as a result of refraction, reflection and other phenomenon [9]. Attenuation is the signal loss associated with frequency dependent absorption in the underwater channel and multiple models exist to estimate the signal attenuation in ocean water. Unlike the geometric spreading and signal attenuation, anomaly of sound propagation is extremely difficult to estimate since it encompasses all losses that might occur due to leaky communication ducts, scattering and diffraction effects that are not already attributed to geometrical spreading or attenuation and effects of depth, temperature, salinity and acidity. The attenuation, in $\mathrm{dB}$, that occurs over a transmission range $l$ for a signal frequency $f$ can be obtained by:

$$
10 \log A(l, f)=k \cdot 10 \log l+l \cdot \alpha
$$

where $\alpha$ is the absorption coefficient in $\mathrm{dB} / \mathrm{km}$ and $k$ represents the geometrical spreading factor. Geometrical spreading loss can be widely categorized as spherical or cylindrical. Spherical spreading occurs when the transmitter and receiver are located a short distance or in deep water, while cylindrical spreading is pronounced in long range communications and shallow water operations due to the surface-bottom reflections of acoustic waves. The geometrical spreading factor can be substituted with values shown in Table I in order to represent the type of spreading that occurs.

\section{Absorption Coefficient}

Attenuation by absorption occurs due to the conversion of acoustic energy within sea-water into heat. This process of attenuation by absorption is frequency dependent since at higher frequencies more energy is absorbed. At low frequencies, the absorption in standard seawater is so small that immense quantities of such water are required to create measurable losses of sound energy into heat. There are several equations describing the processes of acoustic absorption in seawater which have laid the foundation for current knowledge. 
TABLE III

Formulae Providing PSD of THE AMBIENT NoISE

$$
\begin{gathered}
10 \log N_{t}(f)=17-30 \log f \\
10 \log N_{s}(f)=40+20(s-0.5)+26 \log f-60 \log (f+0.03) \\
10 \log N_{w}(f)=50+7.5 w^{\frac{1}{2}}+20 \log f-40 \log (f+0.4) \\
10 \log N_{t h}(f)=-15+20 \log f
\end{gathered}
$$

The attenuation by absorption is dependent upon the ambient conditions, transmission frequency and distance, and as such, the Fisher \& Simmons model proposed in [10] is used for the modeling work presented here. This model also takes into account the effects of relaxation frequencies caused by the presence of boric acid and magnesium sulphate in the ocean:

$$
\alpha=A_{1} P_{1} \frac{f_{1} f^{2}}{f_{1}^{2}+f^{2}}+A_{2} P_{2} \frac{f_{2} f^{2}}{f_{2}^{2}+f^{2}}+A_{3} P_{3} f^{2}
$$

In Equation $3 A_{1}, A_{2}$ and $A_{3}$ represent the effects of temperature on signal absorption, while $P_{1}, P_{2}$ and $P_{3}$ represent the effects of depth and $f_{1}$ and $f_{2}$ represent the relaxation frequencies introduced due to the absorption caused by the presence of boric acid and magnesium sulphate in oceanic water. These coefficients may be obtained from Table II.

\section{Ambient Noise Model}

Ambient noise in the underwater channel has a direct impact upon the quality and dependability of communications, and as such, it is important to model the impact of this phenomenon on acoustic communications as well. Ambient noise in the ocean can be described as Gaussian and having a continuous power spectral density (PSD). The four most prominent sources for ambient noise are the turbulence, shipping, wind driven waves and thermal noise. The overall PSD in $\mathrm{dB}$ re $\mu \mathrm{Pa}$ per $\mathrm{Hz}$ for the combined effects of all these sources can be obtained from the following equation:

$$
N(f)=N_{t}(f)+N_{s}(f)+N_{w}(f)+N_{t h}(f)
$$

The ambient noise in the ocean is colored and hence different factors have pronounced effects in specific frequency ranges. The PSD of each individual source is given by the formulae [11] shown in Table III. The colored effect of noise is represented by $N_{t}$ as the turbulence noise, $N_{s}$ as the shipping noise ( $s$ as the shipping factor lies between 0 and 1), $N_{w}$ as the wind driven wave noise ( $w$ as the wind speed in $\mathrm{m} / \mathrm{s}$ ) and $N_{t h}$ as the thermal noise.

\section{Channel Characterization Model}

The performance of the underwater acoustic channel can be characterized by signal-to-noise ratio (SNR) and received signal power. While the real bit-rate involved in communications would also be handy, this is dependent upon many unmodelable factors and, as such, the channel capacity makes a good metric as well. The equations presented by the authors of [6], [12] are utilized in order to perform the channel characterization in our simulator.

\section{A. Signal-to-noise Ratio}

The signal-to-noise ratio (SNR) of an emitted underwater acoustic signal at the receiver can be expressed by the passive sonar equation [11]:

$$
S N R=S L-T L-N L+D I
$$

Here, $S L$ is the source level, $T L$ is the transmission loss, $N L$ is the noise level and $D I$ is the directivity index. The path loss represented by Equation 2 is the transmission loss $T L$. The noise level $N L$ may easily be obtained from Equation 4, and as such one can evaluate the SNR observed at a receiver over a distance $l$ when the transmitted signal is a tone of frequency $f$ and power $P_{t x}$. Not counting the directivity indices and losses other than the path loss, the narrow-band SNR is, as such, given by:

$$
S N R(l, f)=\frac{P / A(l, f)}{N(f) \Delta f}
$$

where $\Delta f$ is the receiver noise bandwidth, i.e., a narrow band around the transmission center frequency $f$. Once a transmission bandwidth $B$ is chosen for the transmission distance $l$, such that

$$
B=f_{\max }-f_{\min }
$$

around a transmission frequency $f$, the passive sonar equation may be rewritten to obtaining the SNR in $\mathrm{dB}$ :

$$
\begin{aligned}
10 \log S N R(l, f)= & 10 \log P_{t x}-10 \log A(l, f) \\
& -10 \log N(f)-10 \log B
\end{aligned}
$$

Rewriting Equation 8 can be useful in determining the transmission power necessary in order to maintain a minimum SNR for a certain transmission distance and frequency at a particular depth and ambient oceanic conditions.

\section{B. Received Signal Power}

If a signal with frequency $f$ is transmitted over distance $l$ with a power $P_{t x}$ then we can calculate the arriving signal power $P_{r x}$ in $\mathrm{dB}$ :

$$
10 \log P_{r x}=10 \log P_{t x}-10 \log A(l, f)
$$

The result obtained from Equation 9 takes only the case for directional transmission in to account, i.e., the most direct propagation path from transmitter to receiver. However, in case of a transmission that is not directional needs to be modeled, this equation can be extended for the indirect routes as well. 


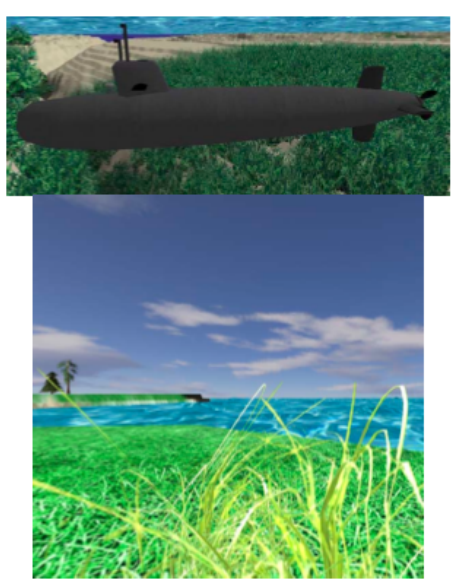

Fig. 1. Screenshot of the USARSim default aquatic model and submarine

\section{Channel Capacity}

The authors of [13] use a channel model with additive Gaussian noise and in [14] the work focuses on using a Rayleigh fading model along with additive white Gaussian noise. The work described in [6], [15] establishes the relationship between capacity and distance, while the authors of [12] show the dependence of capacity on depth and temperature as well.

As such, in order to account for the dependency of capacity on transmission frequency and distance, depth and temperature, we utilize equations proposed by [12]. We utilize the Shannon theorem to determine the maximum capacity bound and by extrapolating from Equation 8:

$$
C(l)=\int_{B} \log _{2}\left(1+\frac{P_{t x}}{A(l, f) N(f) B}\right) d f
$$

where $C(l)$ is the channel capacity for a signal transmitted over a distance $l$.

\section{USARSIM AND UNDERWATER SIMULATIONS}

Based on the Unreal Tournament game engine, USARSim is a high-fidelity simulation tool for simulating robots in complex physical environments. Advanced editing features for almost every aspect of the simulation, further adds to the advantages of USARSim. In this section we provide some details on the underwater environmental and submersible vehicle modeling capabilities of USARSim along with information on WSS and the extensions we made to both these tools in order to enable multi-AUV simulations.

\section{A. Underwater Environments}

In order to correctly evaluate the communication model and test the effects of algorithms, methods and control schemes it is important to have environment and robot models that mimic reality. USARSim has a model world simulating an underwater environment available by default, but others can also be easily created using the Unreal Tournament model editor. We used the default model containing water as our testing world model.

\section{B. Vehicle Model}

Though any vehicle models can be created and imported into the USARSim environment, we chose to use the Submarine model which is provided by default. This model can have sonar sensors, imaging sensors, echo-sounders, side scan and an optical camera simulated on it. The default implementation of the library to interface with USARSim did not have an implementation of a driving mechanism for the submarine and as such we implemented a drive mechanism for the propeller, rudder and stern planes, thereby providing us full mobility control of the submarine and giving us access to testing multi-AUV missions.

\section{Wireless Simulation Server}

WSS is an USARSim plugin that enables simulation of $802.11 x$ wireless network links. WSS works using plugins to implement propagation models allowing for further extensibility. The signal degradation is calculated based upon parameters that are setup for the propagation model plugin and it governs whether connection between robots is possible or not.

Our channel model was implemented as a propagation model plugin for WSS. In our implementation, the user can configure the ambient noise parameters to suit the real environment being modeled. Since USARSim does not have a way to provide the depth of the robot to WSS, a sea level function was implemented to define the sea level in the world map so that the robot's depth could be calculated using its Cartesian coordinates. The determined depth is used to obtain the temperature from the global thermocline average to compute the propagation delay and attenuation coefficient. The user can also specify the signal transmission strength, cutoff strength, bandwidth and center frequency to model any modem without making changes to WSS or USARSim.

WSS by default only supports robots being able to retrieve signal strength at the target robot in order to determine the possibility of communications. This is inadequate for the underwater networking scenario where the ability to retrieve propagation delay and channel capacity is also important. As such, the following functions were implemented within WSS in order to better support the requirements of the underwater environment:

- GETPD returns the propagation delay between the querying robot and the target robot specified in the query string.

- GETBW returns the channel capacity in kbps between the querying robot and the target robot specified in the query string.

The environmental modeling ability of USARSim gives the capability of also modeling and obtaining a bottom profile of the ocean floor. This is helpful since the bottom of the ocean is a great contributor to signal interference as a result of reflections that occur from the seabed in shallow water acoustic communications. The bottom profile can have a significant effect upon multi-path propagation interference as well. As such, it is important to be able to test the 


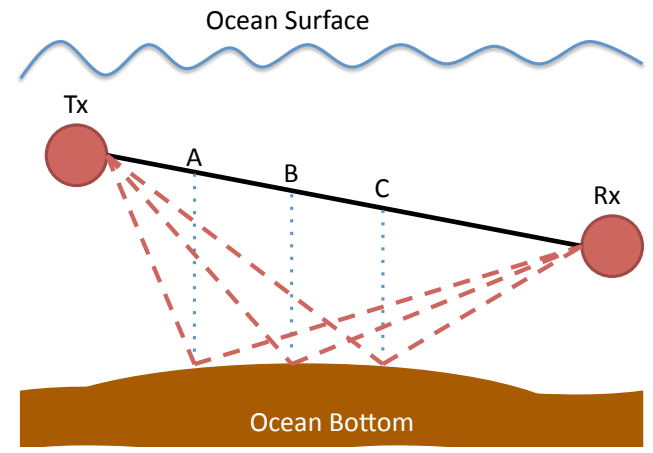

Fig. 2. Multi-path signal propagation in the underwater environment and the use of ray-traces in USARSim \& WSS to retrieve their signal strength at receiver to obtain multi-path interference likelihood.

likelihood of interference due to bottom reflections with the transmission signal. WSS was extended to retrieve bottom multi-path signal interference through the following function:

- GETML returns the interference likelihood as 0 or 1 for a distance to the surface bottom provided in the query string.

In order to use the GETML function the bottom profile between the transmitting and receiving AUV is retrieved as a depth map by performing a ray trace. As shown in Figure 2, the depth of the bottom at multiple points, in this case $A, B$ and $C$, across the direct transmission line between the transmitter and receiver is obtained. Another ray trace between the transmitter, each of the bottom points and receiver is performed in order to obtain the path that a multi-path echo scattered transmitted signal would take, represented by the red lines in Figure 2. The total distance travelled by a reflected wave, from transmitter to bottom and then to receiver, is obtained. This distance for each individual wave is used to determine the arriving signal strength on a particular path by using the channel characterization equations. In case, the PSD of any of the sampled paths is higher than the minimum required reception strength, as determined by the modem properties, multi-path signal interference is determined to be likely.

Though only three points along the direct path are considered in Figure 2, this is only for example. The actual simulator implementation takes the number of points across the direct propagation path to be considered as an input variable set by the GETML command.

WSS performs the channel characterization in real-time, as the simulation is executed within USARSim and provides feedback to the querying robots. This real-time calculation allows the simulation to take in to account any changes that may occur in the environment or any actions the submersible vehicle might be taking at the time.

\section{Simulation Results}

The USARSim simulator provides a familiar environment to develop and perform multi-AUV simulations in. However, before the results of a simulator can be considered dependable, they must be validated. Multiple experiments were run

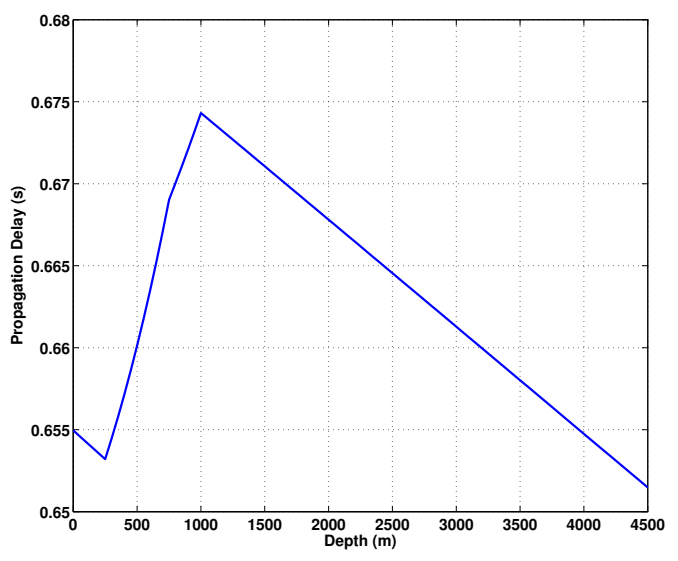

Fig. 3. The change in propagation delay with depth of the two nodes The propagation delay curve follows a shape similar to that of the sound velocity profile.

in order to test the accuracy of the results reported by the simulator.

An experiment similar to the one run by Harris et al. [15] was used to determine the accuracy of propagation delay calculated by the simulator. The depth of two AUVs situated $1 \mathrm{~km}$ apart was progressively increased, while maintaining a constant relative depth for both AUVs. The resulting values of propagation delay are plotted in Figure 3. It is clearly evident that USARSim WSS results mimic those previously reported since the shape of the obtained curve closely mimics that of the sound-velocity profile curve implemented in the simulator.

Quality of an arriving signal is determined using the arriving signal strength, and this information is extremely useful since a signal may only be processed without errors if its power is above the detection threshold of the modem being used. The evaluation of the arriving signal strength is not a straightforward comparison like other values since it is dependent upon the transmission signal strength and the work in published literature is based upon the transmission strength necessary to achieve a desired SNR. As such, in order to test the accuracy of the simulator, it is important to draw a few inferences from available data.

The relationship between capacity and distance is well established. As such, it is widely accepted that available capacity reduces exponentially with distance. Conversely, higher transmission power is necessary to achieve a higher capacity [12] . Therefore, available capacity is proportional to the transmission power utilized. Thus, we can deduce that the signal strength should reduce with distance in a somewhat logarithmic fashion, with the curves closely following the shape of the capacity curves.

Keeping this in mind an experiment while keeping a depth of $100 \mathrm{~m}$ constant for two AUVs, using the standard thermocline and varying the distance between both the AUVs from $4 \mathrm{~m}$ to $180 \mathrm{~m}$ was used. The transmission power was also varied between $60 \mathrm{~dB}$ and $120 \mathrm{~dB}$. The results of this 


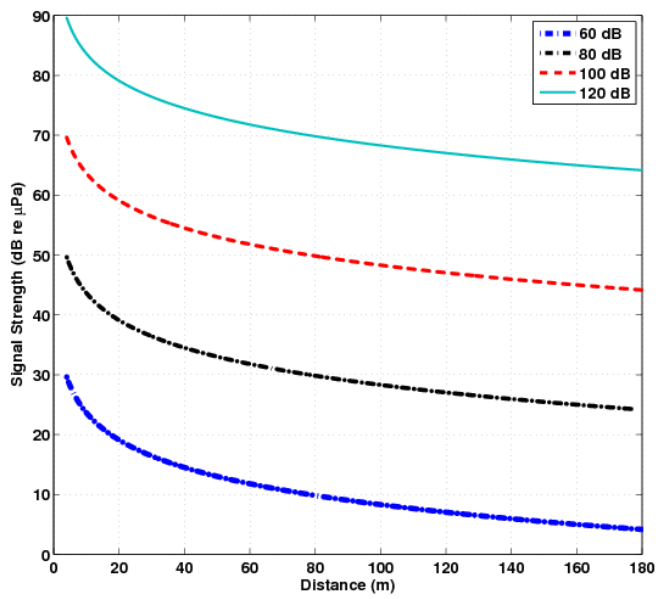

Fig. 4. The arriving signal strength while the distance between the transmitting and receiving nodes was varied between 4 to $180 \mathrm{~m}$ and the transmit power is also changed.

experiment performed in the USARSim WSS environment can be seen in Figure 4. Since the shape of this figure follows the expected shape, i.e. an exponential reduction in signal strength with increasing distance, it can be deduced that the simulator works accurately. However, even further confirmation of the accuracy of these results is forthcoming via the capacity results.

Using the same experiment as that for received signal power, the values of channel capacity were also obtained and plotted in Figure 5. It is clear from Figure 5 that the capacity reduces with distance between the nodes and increases with increased transmission power. The similarity of the shape of the curves to those obtained by numerical evaluation by Stojanovicet al [6] establishes the accuracy of the simulator with respect to capacity as well. Furthermore, the similarity of the capacity and signal strength curves also shows the close relationship between these two values in the underwater acoustic channel.

\section{CONCLUSIONS}

Propagation models for the underwater acoustic channel were implemented within the framework of the USARSim robotics simulator by extending the WSS plugin. This implementation enables the simulation of underwater acoustic communications, in support of multi-AUV simulations. The implemented provide results which are as close to off-shore performance as possible. The calculation of a multi-path interference likelihood based upon bottom profile is unique to our implementation and provides a feature that could help researchers build systems which minimize effects of this phenomenon.

The implementation of underwater acoustic channel models within the USARSim-WSS framework provides an effective simulation tool for multi-AUV missions.

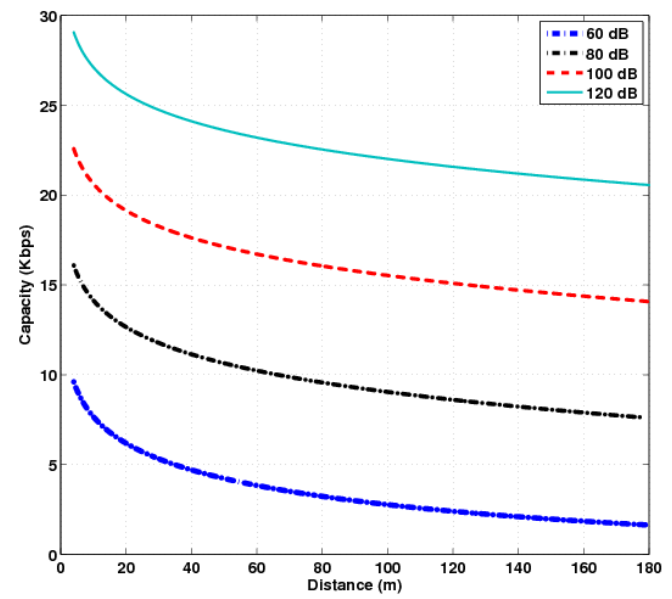

Fig. 5. The channel capacity while the distance between the transmitting and receiving nodes was varied between 4 to $180 \mathrm{~m}$ and the transmit power is also changed.

\section{REFERENCES}

[1] I. F. Akyildiz, D. Pompili, and T. Melodia, "Challenges for efficient communication in underwater acoustic sensor networks," SIGBED Rev., vol. 1, no. 2, pp. 3-8, 2004.

[2] J. Partan, J. Kurose, and B. N. Levine, "A survey of practical issues in underwater networks," in WUWNet '06: Proceedings of the 1st ACM international workshop on underwater networks, (New York, NY, USA), pp. 17-24, ACM, 2006.

[3] E. Sozer, M. Stojanovic, and J. Proakis, "Underwater acoustic networks," IEEE Journal of Oceanic Engineering, vol. 25, pp. 72-83, January 2000.

[4] J. Craighead, R. Murphy, J. Burke, and B. Goldiez, "A survey of commercial \& open source unmanned vehicle simulators," in 2007 IEEE International Conference on Robotics and Automation, 2007.

[5] T. Bielohlawek, "Subsim - an autonomous underwater vehicle simulation system," Master's thesis, Universitaet Kaiserslautern, 2006.

[6] M. Stojanovic, "On the relationship between capacity and distance in an underwater acoustic communication channel," in WUWNet '06: Proceedings of the 1st ACM international workshop on underwater networks, (New York, NY, USA), pp. 41-47, ACM, 2006.

[7] K. V. MacKenzie, "Nine-term equation for sound speed in the oceans," Acoustical Society of America Journal, vol. 70, pp. 807-812, Sept. 1981.

[8] A. Caiti, E. Crisostomi, and A. Munafo, "Physical characterization of acoustic communication channel properties in underwater mobile sensor networks," in Proceedings of International Conference on Sensor Systems and Software, (Pisa, Italy), September 2009.

[9] H. G. Urban, Handbook of Underwater Acoustic Engineering. STN ATLAS Elektronik GmbH, November 2002.

[10] F. H. Fisher and V. P. Simmons, "Sound absorption in sea water," The Journal of the Acoustical Society of America, vol. 62, no. 3, pp. 558564, 1977.

[11] R. J. Urick, Principles of Underwater Sound. Los Altos, California: Peninsula Publishing, third ed., 1983.

[12] A. Sehgal, I. Tumar, and J. Schoenwaelder, "Variability of available capacity due to the effects of depth and temperature in the underwater acoustic communication channel,' in Proc. of IEEE OCEANS '09, (Bremen), May 2009.

[13] H. Kwon and T. Birdsall, "Channel capacity in bits per joule," IEEE Journal of Oceanic Engineering, vol. 11, no. 1, pp. 97-99, 1986.

[14] H. A. Leinhos, "Capacity calculations for rapidly fading communications," IEEE Journal of Oceanic Engineering, vol. 21, no. 2, pp. 137$142,1996$.

[15] A. F. Harris and M. Zorzi, "Modeling the underwater acoustic channel in ns2," in ValueTools '07: Proceedings of the 2nd international conference on Performance evaluation methodologies and tools, (ICST, Brussels, Belgium, Belgium), pp. 1-8, ICST, 2007. 